\title{
PROGNÓSTICO DE TUMORES TESTICULARES GERMINATIVOS ${ }^{1}$
}

\section{OUTCOME OF GERM CELL TUMORS}

\author{
José Anastácio Dias Neto ${ }^{2}$ \\ André Luiz Alonso Domingos ${ }^{2}$ \\ Antonio Carlos Pereira Martins ${ }^{3}$ \\ Silvio Tucci Jr. ${ }^{3}$ \\ Haylton Jorge Suaid ${ }^{3}$ \\ Adauto José Cologna ${ }^{3}$ \\ Cassio Botene Schneider ${ }^{4}$
}

\section{RESUMO}

Objetivo - Investigar as características e a evolução de homens adultos portadores de tumores germinativos do testículo. Métodos - Estudamos as características e a evolução 29 pacientes tratados (14 seminomas e 15 não seminomas). O tempo médio de seguimento foi de 56 meses para os seminomas e de 40 meses para os não seminomatosos. Todos foram submetidos a orquiectomia. Nos estádios II e III associou-se radioterapia para os seminomas, e quimioterapia e linfadenectomia para os não seminomatosos. Resultados - As queixas mais freqüentes foram aumento de volume testicular (57\%) e dor (30\%). Nos seminomas a idade média foi de 41,2 anos e nos não seminomas foi de 29,2 anos. Antecedente de criptorquidia foi assinalada em $28,5 \%$ dos seminomas e em $15,5 \%$ dos não seminomatosos. As proporções respectivas de estádios I, II e III foram de 79\%, 14\% e $7 \%$ em seminomas, e $40 \%, 27 \%$ e $33 \%$ em não seminomas. Os seminomas não provocaram elevação dos marcadores AFP ou b-HCG enquanto os não seminomatosos elevaram esses marcadores respectivamente em 46,6\% e 33,3\% dos casos. Morte pela doença ocorreu em 1 caso de seminoma e 3 de não seminomas, mas não houve diferença na sobrevida entre os grupos. Conclusão - A criptorquidia continua sendo um fator predisponente importante na etiologia dos tumores germinativos. Apesar dos tumores não seminomatosos se apresentarem em estádios mais avançados a sobrevida dos pacientes não difere da apresentada pelos portadores de seminomas.

Disponível em URL: http://www.scielo.br/acb

Descritores - tumor testicular, tumor germinativo, seminoma, não seminoma,

\section{ABSTRACT}

Objective - The aim of the study is to analyze the characteristics and the evolution of patients with testicular germ cell tumors. Methods - We analyzed 29 patients: 14 seminomas and 15 non-seminomas. All of them underwent orquiectomy. Patients with seminomas stage II and III received adjuvant treatment with raditherapy, and those with non-seminomas stage II and III received neoadjuvant chemotherapy followed by lymphadenectomy. Mean followup for seminomas was 56 months and for non-seminomas it was 40 months. Results - The most common complain was an increase in testis volume (57\%) and pain (30\%). The mean age in seminomas was 41.2 years and in non-seminomas it was 29.2 years. Previous criptorquidy was refered by $28.5 \%$ of patients with seminomas and $15.5 \%$ with non-seminomas. The respective proportions of stages I, II and III were 79\%, $14 \%$ and $7 \%$ in seminomas, and $40 \%, 27 \%$ and $33 \%$ in non-seminomas. Patients with seminomas did not show serum rise of alfa-fetoprotein or b-HCG while those with non-seminomas such tumor markes were elevated respectively in $46.6 \%$ and $33.3 \%$ of the sample.

Trabalho realizado na Divisão de Urologia, HCFMRP-USP

2 Médicos Residentes da Divisão de Urologia, HCFMRP-USP

3 Docentes da Faculdade de Medicina de Ribeirão Preto - USP

4 Pós-graduando da FMRP-USP 
Disease specific death occurred in 1 patient with seminoma and in 3 with non-seminoma tumor, but survival curves were similar for both groups. Conclusion - In spite of the earlier treatment of criptorquidy such an antecedent stand as an important risk factor for the development of testicular germ cell tumor. Even though non-seminomas presents with higher stages the survival curves are similar for both groups of tumors. Analyze 29 patients that were submitted to orquiectomy (14 seminomas e 15 non seminomas), concerning their age, signs and symptoms, risk factors, clinical staging, tumor markers follow up and survival rates. Results - There was no statistically difference in survival rates of patients with seminomas and non-seminomas tumors.

Key Words - testicular tumor, seminoma, nonseminoma, germ cell tumor.

\section{INTRODUÇÃO}

O câncer de testículo, apesar de raro, é a neoplasia maligna mais comum entre homens jovens entre $15 \mathrm{e}$ 35 anos $^{1}$. Os avanços no estudo das neoplasias testiculares nos últimos anos, refletem-se no aumento dos índices de cura da doença mesmo em casos avançados. Com o emprego da quimioterapia, radioterapia e cirurgia, os índices de sobrevida em 5 anos aumentaram de $50 \%$ na década de 60 para $90 \%$ atualmente.

Os tumores primários do testículo são divididos em germinativos e não germinativos. Os tumores germinativos, que incluem os seminomas, não-seminomas (carcinoma embrionário, teratoma, teratocarcinoma, coriocarcinoma e tumores do saco vitelino) e os tumores mistos, correspondem a $90 \%$ das neoplasias testiculares e a $0,5 \%$ das neoplasias do sexo masculino. Tem menor incidência na raça negra. O seminoma é mais freqüente entre a população adulta (45 a $50 \%$ dos casos). Já na população infantil, o tumor germinativo mais comum é o tumor do saco vitelino $(80 \% \text { dos casos) })^{2}$.

Os tumores não germinativos são muito mais raros e correspondem a apenas 2 a 3\% das neoplasias testiculares, compreendem os tumores de células de Leydig, tumores de células de Sertoli, gonadoblastomas e mistos ${ }^{1,2,3}$.

Não foi possível identificar fatores de risco ambientais, ocupacionais ou virais, porém há associações causais com fatores genéticos, hormonais, congênitos (criptorquidia), e adquiridos (atrofia e trauma testi(cular) $)^{4}$.

Os tumores germinativos não seminomatosos $(85 \%)$ e os seminomatoos (10\%) apresentam capacidade de sintetizar glicoproteínas que são utilizados como marcadores tumorais (gonadrofina coriônica humana
-hCG e alfa fetoproteína-AFP e LDH). O hCG e AFP estão relacionados com a atividade do tumor, e os níveis de LDH correlacionam-se com o volume tumoral ${ }^{4,5}$.

O objetivo do presente estudo é analisar algumas características e o prognóstico dos tumores germinativos testiculares em adultos.

\section{MÉTODOS}

Revisamos os prontuários de pacientes submetidos a orquiectomia por neoplasia testicular no Hospital das Clínicas da Faculdade de Medicina de Ribeirão Preto da Universidade de São Paulo- HC-FMRP-USP no período de 1975 à 2002.

Encontramos 42 pacientes: 29 (70,7\%) adultos com tumores germinativos, 1 adulto com câncer metastático de estômago, 5 crianças com tumores do saco vitelino, 04 crianças com tumores estromais e 02 com rabdomiossarcoma, $01 \mathrm{com}$ linfoma. No estudo forma incluídos apenas os 29 pacientes adultos com tumores germinativos.

Classificamos os tumores germinativos em seminomatosos [n=14 (48,2\%)] e não seminomatosos $[\mathrm{n}=15(51,7 \%)]$. Empregou-se o método de Boden \& Gibb $^{6}$ para o estadiamento.

O tratamento dos seminomas consistiu em orquiectomia e radioterapia abdominal (estádio I). No estádio II acrescentou-se radioterapia mediastinal e fossas supraclavivulares. No estádio III empregou-se orquiectomia associada à quimioterapia $\left(\mathrm{VAB}_{6}\right)$. Os tumores não seminomatosos no estádio I foram tratados com orquiectomia; a linfadenectomia retroperitoneal associada foi usada somente em pacientes não confiáveis e nos casos de maior risco (invasão do cordão ou epidídimo, invasão vascular, carcinoma embrionário puro e exame de imagem retroperitoneal duvidoso). Quando no estádio II e III associou-se a linfadenectomia retroperitoneal e quimioterapia( $\mathrm{VAB}_{6}, \mathrm{PVB}$ ou $\left.\mathrm{PEB}\right)$.

$\mathrm{O}$ método de Kaplan-Meier foi utilizado para comparar a sobrevida dos pacientes com tumores seminomatosos daqueles com tumores não seminomatosos, e o teste logrank ${ }^{11}$ para comparar as curvas de sobrevida, através do programa Graph Prism, versão 6.07,8. Considerou-se o tempo de 24 meses de seguimento como ponto de corte.

\section{RESULTADOS}

As queixas mais freqüentes entre os pacientes foram aumento de volume testicular (57\%) e dor (30\%).

Entre os pacientes com tumores seminomatosos, a idade variou de 24 a 60 anos com média de 41,2 $(\mathrm{SD}=10,37)$. O seguimento médio foi de 56 meses (6- 
120 meses). Ambos os lados eram acometidos na mesma proporção (Tabela 1). A presença de criptorquidia foi observada em 3 casos dos tumores seminomatosos (21\%), atrofia testicular em 01 caso $(7,5 \%)$ e 01 caso com atrofia e criptorquidia (7,5\%). O estádio na apresentação foi: $11(79 \%)$ tumores apresentavam estádio I, 2 (14\%) estádio II e 1 (7\%) estádio III (Tabela 2).

Tabela 1 - Características clínicas dos pacientes com tumores germinativos.

\begin{tabular}{lcccc}
\hline \multicolumn{1}{c}{ Tipo } & N & Idade média \pm SD & Seguimento (meses)* & Proporção D:E** \\
\hline Seminoma & 14 & $41,2 \pm 10,3$ & 56 & $1: 1$ \\
Não seminoma & 15 & $29,6 \pm 11,2$ & 40 & $4: 1$ \\
\hline
\end{tabular}

* Médio; ** $\mathrm{D}=$ testículo direito e $\mathrm{E}=$ testículo esquerdo

Tabela 2 - Estadiamento segundo o tipo histológico.

\begin{tabular}{lccc}
\hline \multirow{2}{*}{ Tumor germinativo } & \multicolumn{3}{c}{ Estádio } \\
\cline { 2 - 4 } & I & I I & III \\
\hline Seminoma & $11(79 \%)$ & $2(14 \%)$ & $1(7 \%)$ \\
Não seminoma & $6(40 \%)$ & $4(27 \%)$ & $5(33 \%)$ \\
\hline
\end{tabular}

No grupo de pacientes com tumores não seminomatosos, o carcinoma embrionário foi o tumor mais freqüente $(53,4 \%, \mathrm{n}=8)$, seguido pelo tumor misto $(20 \%, n=3)$, teratoma $(13,3 \%, n=2)$ e teratocarcinoma $(13,3 \%, \mathrm{n}=2)$. A idade dos pacientes variou entre $18 \mathrm{e}$ 41 anos, com média de 29,6 ( $\mathrm{SD} \pm 11,2$ ). O tempo médio de seguimento foi de aproximadamente 40 meses (1240 meses). Notamos maior acometimento tumoral no testículo direito, na proporção de 4:1 (Tabela 1). Dois pacientes $(15,5 \%)$ apresentaram criptorquidia e em 01 paciente $(7,7 \%)$ houve história de trauma prévio. Ao diagnóstico, 6 (40\%) tumores apresentavam Estádio I, 4 (27\%) Estádio II e 5 (33\%) com Estádio III.

Analisando a presença de marcadores tumorais nos pacientes com tumores seminomatosos, encontramos cinco casos $(35,7 \%)$ com aumento significativo de LDH, no entanto, b HCG e a feto proteína não se apresentaram aumentados.

O LDH estava aumentada em 6 (40\%) pacientes com tumores não seminomatosos, a a-feto proteína em $7(46,6 \%)$ e ob-HCG em dois pacientes $(13,3 \%)$.

Tabela 3 - Marcadores tumorais segundo o tipo histológico.

\begin{tabular}{lccc}
\hline \multicolumn{1}{c}{ Tipo histológico } & AFP* & B HCG*** & LDH*** \\
\hline Seminoma & $0 \%$ & $0 \%$ & $37,7 \%$ \\
Não seminoma & $46,6 \%$ & $13,3 \%$ & $40 \%$ \\
\hline
\end{tabular}

* Alfa-fetoproteina, ** Fração $ß$ da gonadotrofina coriônica, *** Desidrogenase láctica

Não houve diferença na sobrevida entre pacientes com tumores seminomatosos e não seminomatosos, $\mathrm{p}=0,7$ (Figura 1).

Perderam seguimento $14,2 \%(\mathrm{n}=2)$ dos pacientes com tumores seminomatosos. Em 01 caso $(7,1 \%)$ ocorreu óbito (estádio II ao diagnóstico).
Em $2(13,2 \%)$ pacientes com tumores não seminomatosos perdeu-se o seguimento. Três pacientes $(20 \%)$ faleceram, sendo 2 com estádio III e 01 com estádio II, sendo que o intervalo entre o diagnóstico e óbito foi de 1, 3 e 13 meses, respectivamente. 


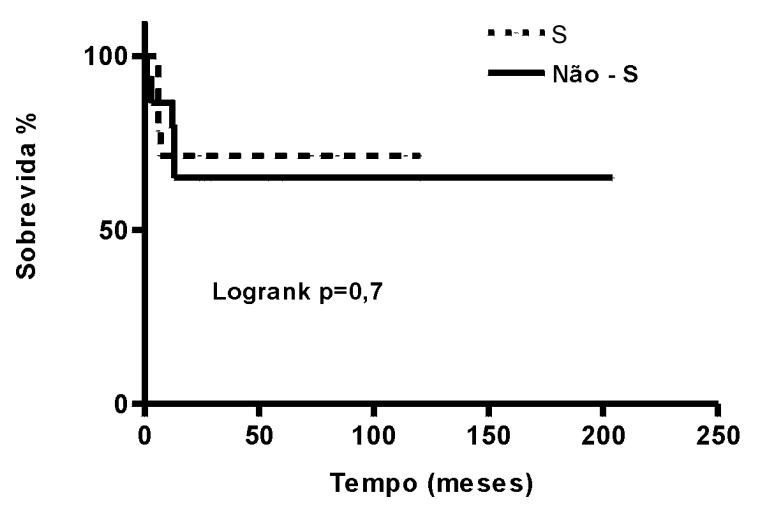

Figura 1 - Curva de Kaplan Méier comparando seminomas (S) e não seminomas (Não-S).

\section{DISCUSSÃO}

Os tumores germinativos foram os mais freqüentes entre as neoplasias testiculares. A maior incidência dos tumores testiculares entre jovens está bem descrita na literatura ${ }^{1}$. Os tumores seminomatosos corresponderam à $48,2 \%$ dos tumores germinativos, o que é relatado para outras séries ${ }^{1,2,9}$.

Apesar da conduta de se corrigir precocemente a criptorquidia este antecedente continua a representar um fator de risco. A criptorquidia foi o fator mais consistentemente associado ao tumor testicular, sendo encontrada em $28,5 \%$ dos seminomas e $15,5 \%$ dos não seminomatosos. Essas proporções são superiores às relatadas em ourtos artigos ${ }^{4,5}$. $\mathrm{O}$ aumento de volume do testículo foi o principal sintoma encontrado, entretanto a dor aguda testicular foi observada com maior freqüência ( $30 \%$ dos casos) que outros estudos ${ }^{10}$.

A LDH, produzido por todos os tipos de tumores testiculares e relacionada com o volume tumoral ${ }^{11,12}$ apresentou-se elevada em 35,7\% dos seminomas e 40\% dos não seminomas. Embora haja referências de que $8 \%$ dos seminomas produzão b $\mathrm{HCG}^{13}$, em nossos pacientes os tumores seminomatosos não produziram b HCG e a feto proteína. Já a proporção de tumores não seminomatosos produtores desses marcadores foi um pouco inferior ao relatado anteriormente ${ }^{13}$.

Não encontramos diferença entre a sobrevida de pacientes com tumores seminomatosos dos não seminomatosos, o que se deve sobretudo à eficácia da quimiterapia como já referida por outros ${ }^{12}$.

\section{CONCLUSÃO}

Os tumores germinativos representam $70 \%$ dos tumores testiculares. A queixa clínica mais comum foi o aumento de volume testicular, o fator de predisponente mais comum foi a criptorquidia. A maioria dos pacientes apresentou estádio I ao diagnóstico.

Os pacientes com tumores germinativos não seminomatosos apresentaram estádios mais avançados da doença quando comparados aos seminomatosos, porém não houve diferença na sobrevida ao se comparar ambos os grupos.

\section{REFERÊNCIAS}

1. Ries LAG, Miller BA, Hankey BF. SEER cancer statistics review: 1973-1991- tables and graphs (NIH pub. No.94-2789). Bethesda, MD: National Cancer Institute, 1994.

2. Mostofi FK, Sesterhenn IA, Davis CJ. Immunopathology of germ-cell tumors of the testis. Semin Diagn Pathol 1987;4:320-41.

3. Jacobsen GK, Henriksen OB, Von Der Maase H. Carcinoma in situ of testicular tissue adjacent to malignant germ-cell tumors: a study of 105 cases. Cancer 1981;47:2660-2.

4. Pottern LM, Goedert JJ. Epidemiology of testicular cancer. In: Javadpour $\mathrm{N}$, ed. Principles and management of testicular cancer. New York: Thieme. 1986. p.108-19.

5. Henderson BE, Ross RK, Pike MC. Epidemiology of testicular cancer. In: Skinner DG, Lieskovsky G. Genitourinary cancer. Philadelphia: Saunders, 1988. p.46-51.

6. Boden D, Gibb R. Radiotherapy and testicular neoplasms. Lancet 1951;2:1195-7.

7. Peto R, Pike MC, Armitage P, Breslow NE, Cox DR, Howard SV, Mantel N, McPherson K, Peto J, Smith PG. Design and analysis of randomized clinical trials requiring prolonged observation of each patient. II. Analysis and examples. Br J Cancer 1977;35:1-39.

8. Simon RM. Design and conduct of clinical trials. In: DeVita VT Jr., Hellman S, Rosemberg SA. Cancer principles and pratice of oncology. Volume 1. $3^{\text {rd }}$ edition. Philadelphia : Lippincott. 1989.396-420.

9. Smith RB. Testicular seminoma. In: Skinner DG, Lieskovsky G. Genitourinary cancer. Philadelphia: Saunders, 1988 . p.508-25.

10. Bosl GJ, Vogelzang NJ, Fraley EE et al. Impact of delay in diagnosis on clinical stage of testicular cancer. Lcet, n 2;970, 1981 .

11. Cummings KM, Lampone D, Mettlin C, Pontes JE. What young men know about testicular cancer. Prev Med 1983;12:326.

12. Ernstoff MS, Heaney JA, Peschel RE. Urologic Cancer. Blackwell Science. 541.

13. Javadpour $\mathrm{N}$. The role of biologic tumor markers in testicular cancer. Cancer 1980;45:1755-9.

\section{Endereço para correspondência}

Antonio Carlos Pereira Martins

Faculdade de Medicina de Ribeirão Preto - USP

Departamento de Cirurgia

Av. Bandeirantes $n^{\circ}$ 3900, Ribeirão Preto, SP,

CEP: 14048-900.

e-mail - acpmartins@convex.com.br 